\title{
The Game Theory Application in the Construction Safety Management BuXiangyi
}

Wuhan University of Technology,Wuhan 430070,China

Key words: game analysis; safety management; construction project; Vensim software; visualization

\begin{abstract}
In order to study the influence of the participating parties in the construction process, the construction safety management analysis model based on the static and evolutionary game theory is put forward. The completely static game theory method of construction safety construction units, and supervision units were analyzed, obtained the relationship between the two sides of the basic game theory, the evolutionary game theory to establish the game model of the safety supervision units and construction units, has been on both sides of the balance relationship of strategy. Finally through the Vensim software established construction safety supervision game model of SD, and on safety supervision units and construction units on both sides of the implementation strategy of visual analysis, the safety rules of the construction unit execution probability curve, provide more valuable reference for the research of construction safety management.
\end{abstract}

\section{Introduction}

The construction industry is the pillar industry of our country, and it plays an important role in the economic and social development of our country. The frequent occurrence of building safety accidents is a serious impediment to the economic and social development of our country. Although the current construction safety management rules and system is relatively perfect, but construction engineering safety accident happened frequently, how to effectively control construction safety accidents and improve the economic benefit of construction enterprises, construction safety management needs to focus on the problem. This study using game theory, the analysis of structure in the relationship between the parties, from deep angle excavation construction safety accident causes, which can effectively develop safety management measures, reduce the probability of construction engineering safety accident.

\section{Construction safety management based on Game Theory}

Construction safety supervision units and construction units and owners each have different interests, in order to obtain the maximization of their own interests, the game between the parties will affect the quality of engineering construction, and influence the security of the whole project, the game between the node constitutive relations such as shown in Figure 1. 


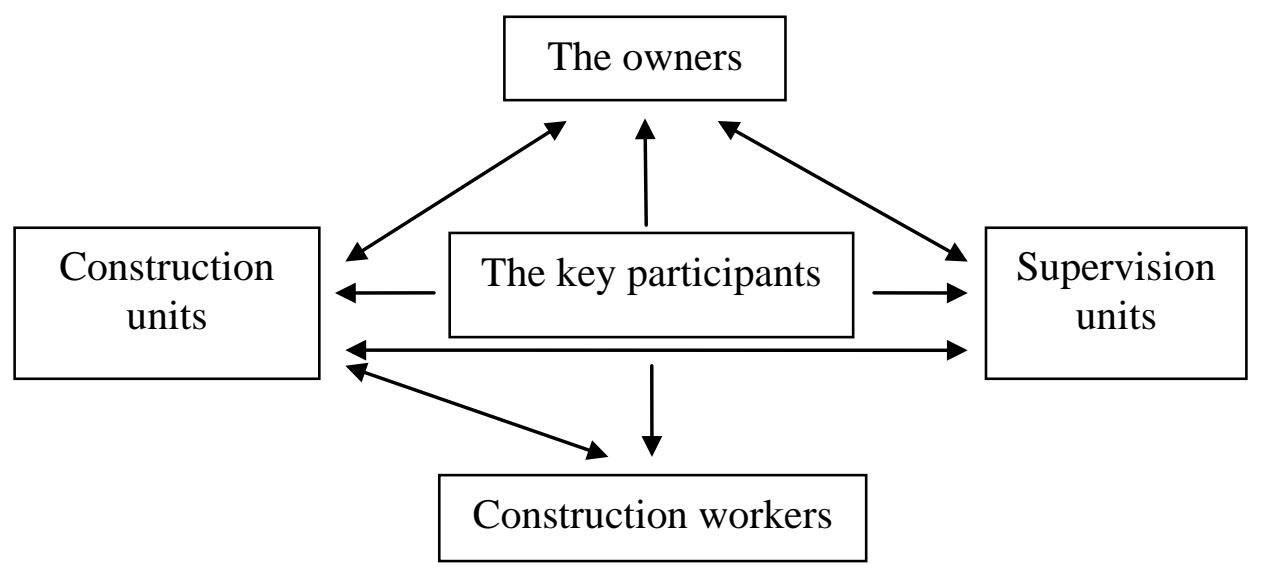

Fig. 1 the game relation structure of construction project participants

From Figure 1 we can see that the relationship between participants in the construction project will affect the engineering safety and quality, the owners and the construction unit is mainly reflected in the interests and objectives of the owners reflect in exhaustion may cost less get high quality engineering, and the construction unit goals is to reduce the construction cost and increase the profit of the project. However, the owners of the payment of funds and contract management has obvious advantages, the construction unit to a disadvantage, in the performance of the contract is likely to cause conflicts of interest, thereby affecting the quality of construction, resulting in security risks. Due to the owners to pay the right to pay, so in the bidding process in the active position, while the construction unit is in a passive position. But the construction units in the construction quality and the technical scheme occupies initiative, in order to pursue the maximization of benefits, the construction units can only rely on drill earnings and anchored and subcontracting profit, including Jerry has become the unspoken rules of the construction industry. At the same time, the owner commissioned the supervision unit to carry out the project supervision, improve the quality of the project, and ensure the safety of the project.

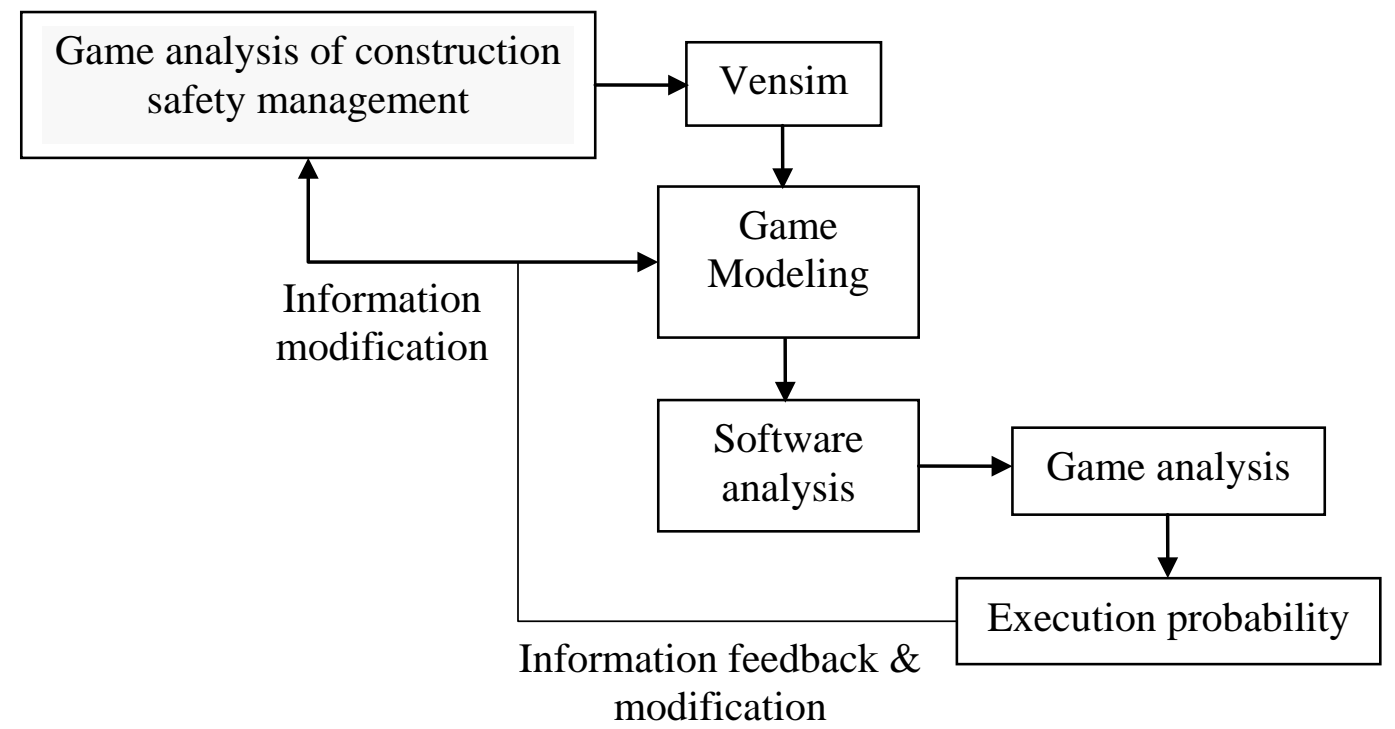

Fig. 2 The game analysis process of construction safety management

Figure 2 the construction safety management of the game analysis between the flow chart, first using Vensim software game relationship model can be established, then the execution probability of dynamic evolution curve is obtained by using software visualization, by probability of the implementation of the strategy. Analysis the game relationship between the parties. 


\section{Game analysis on construction safety management accident case}

A construction company for the construction of a stadium, and commissioned construction supervision company supervision, but in pouring to the cap, the zenith of the stadium collapsed, resulting in six people were killed, 1 seriously injured and 33 people were slightly injured, the economic losses reached more than 70 million, is after the founding of the people's Republic of China the city the largest architectural engineering accident. Stadium collapse and the cause of the accident is mainly due to the supervision units supervision and construction unit of the organization caused confusion. Under such conditions, accident of the main responsible party for the construction and supervision units. Among them, collapse causes including construction unit is strictly in accordance with the safety regulations of the organization construction, supervision unit is to the construction unit of strict supervision.

Hypothesis 1: the safety supervision units of construction engineering company is very good ethics and credibility of the supervision team, from the beginning to the end, did not accept bribes from aspects of the construction, and the incentive and supervision mechanism, establish the supervisor responsibility system, so as to safeguard the interests of the owners of the country; excellent technology of safety supervision units, if there is quality problem of the project will to check.

Hypothesis 2: there is no collusion between the security building units and the construction units, the two sides are a non cooperative game relationship.

Hypothesis 3: the safety supervision unit and the construction unit are the two limited Bureau, whose benefit maximization is the main goal of promoting the movement.

According to the stadium collapse accident can be summed up, the safety supervision unit and construction unit that the interests of the problem is not consistent, through the static game can choose the strict supervision of the critical probability

$$
y^{*}=\frac{c-f l}{m+c}
$$

In the formula (1), $c$ is the investment cost of the construction, $f$ is the probability of the accident, $l$ is the accidents caused by the loss, construction not in accordance with the construction safety rules, fine $m$ found in supervision units, the strictly critical probability that construction units can choose is shown as formula (2).

$$
x^{*}=1-\frac{h-s}{m+f n}
$$

In the formula (2), $s$ is the profits that Security supervision units can get in accordance with the normal supervision, $n$ is Fine of supervision units after the accident, $h$ is the safety supervision cost savings when construction side in accordance with the safety procedures. According to the analysis of relevant data, the cost of the project is 600 million, and the actual input to safety in production cost of only 2 million, can profit from 0.2 million, if in accordance with the safety regulations and construction, safety supervision units will be to the construction unit of 6 yuan fine, the probability of the accident is $6 \%$, the accident unit will be the loss of 6 million yuan, and be fined up to 6 million yuan, and safety supervision unit will be fined 6 million yuan, the construction unit strictly critical probability, $x^{*}$ is closed to 0 .Therefore, the amount of fines will have a greater impact on $x^{*}$, and $\mathrm{m}$ value is greater, the construction unit of the execution probability of the smaller, safety accident occurs more easily, and increasing fines for $m$ and $n$ values, can effectively improve the construction of the security level, so as to improve the efficiency of safety supervision.

Similarly, according to the safety supervision units strict critical probability, if $m$ value is greater, $f$ and $c$ value is small, the probability of safety supervision close to 0 , will cause the supervision unit management, and the construction unit does not execute the safety rules, so as to form a vicious circle. 
But in the actual construction process, human resources are limited, so the $x^{*}$ value is not very large, because the safety supervision is not in place, the construction unit operation violation is inevitable. Therefore, the reasonable allocation of the amount of fines and the cost of supervision of construction is a dynamic trade-off problem, and the strict critical probability can achieve the optimal allocation of this dynamic equilibrium, the expression is the formula (3).

$$
\begin{aligned}
& F(x)=d x / d t=x(1-x)(f l+(m+c) y-c) \\
& F(y)=d y / d t=y(1-y)[(m+s+f n-h)-x(m+f n)]
\end{aligned}
$$

In the formula (3), The five equilibrium point of the equation are $(0,0),(1,0),(1,1),(0,1),\left(\frac{m+f n+s-h}{m+f n}, \frac{c-f l}{m+c}\right)$, and $0 \leq \frac{m+f n+s-h}{m+f n} \leq 1,0 \leq \frac{c-f l}{m+c} \leq 1$.

We can get the Jacobi matrix through the analysis,

$$
J=\left[\begin{array}{cc}
(1-2 x)(f l+(m+c) y-c) & x(1-x)[(m+c)+f l] \\
-y(1-y)(m+f n) & (1-2 y)[(m+f n+s-h)-x(m+f n)]
\end{array}\right]
$$

In the formula (4),

$$
\operatorname{det} J=[(1-2 x)(f l+(m+c) y-c)](1-2 y)[(m+f n+s-h)-x(m+f n)]
$$

$$
\begin{aligned}
& +[(m+c)+f l] x(1-x) y(1-y)(m+f n) \\
& \operatorname{tr} J=[(1-2 x)(f l+(m+c) y-c)]+(1-2 y)[(m+f n+s-h)-x(m+f n)]
\end{aligned}
$$

We found that the value of $c$ than $f l$ after the substitution of known data equilibrium point without stable value, so not get stabilizing selection strategy, the implementation of safety supervision units and construction units is with a certain probability.

In the pure mathematical point of view, a reasonable increase penalties for safety supervision and construction unit, increase supervision units, the construction unit of the punishment, reduce the construction unit of the strict implementation of the safety regulations for the critical probability can promote the safety level of the construction unit of the construction, so as to ensure the safety of the whole project. Through the management of safety, can effectively reduce the probability of the accident, reduce casualties and economic losses, from overall situation can better complete safe construction task, this is the best proof of construction safety and game theory.

\section{Construction safety supervision SD game model simulation}

In order to more intuitive descript safety supervision units and construction units of the game, and maintain the stability of behavioral strategies, this study takes the construction unit as an example using Vensim software construction safety supervision of SD game model is established. Vensim is a visual simulation modeling software, which can describe the dynamic structure of the system, also have the behavior change of the simulation system, and the simulation results are visualized analysis and optimization. Safety supervision units of supervision and the proportion of the construction unit safety regulations for the proportion of execution is dynamic evolution in different conditions. Therefore, it is necessary to game analysis of uncertain factors, the simulation analysis of the frame as shown in Fig.3. 


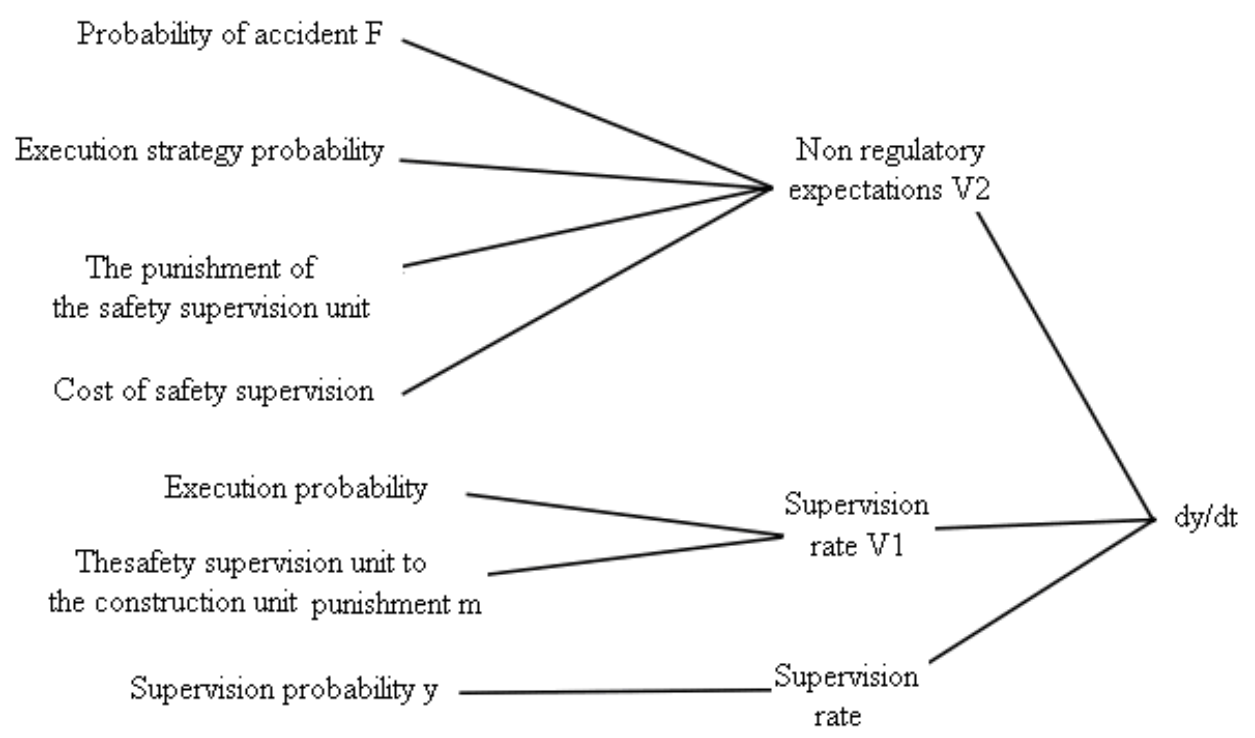

Fig.3 The tree diagram of the increment of supervision probability

Before modeling, we first need to the elements of the system in-depth analysis, interaction relation between the elements, constructing the causal structure of the SD model of construction safety supervision, through the simulation calculation can be obtained execution probability versus time curve as shown in Figure 4.
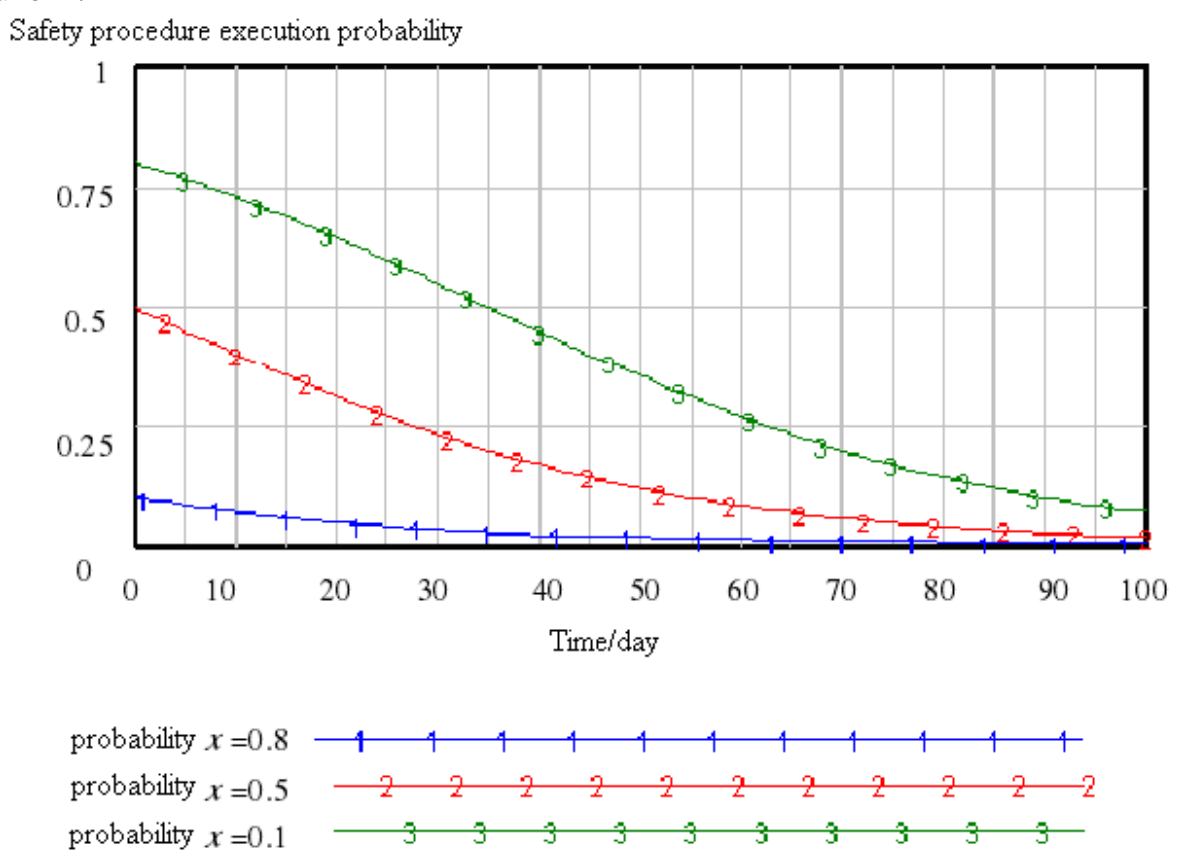

Fig. 4 Dynamic change curve of construction strategy

As is shown in Fig 4, when probability y of the safety supervision unit selection supervision is less than 0.5 , under the condition of different execution probability, probability of execution of the construction unit according to the safety rules eventually converge to 0 , the construction unit will eventually not in accordance with the safety regulations for the implementation of measurement, and the convergence speed with the increase of the initial probability of smaller.

\section{Conclusions}

The construction units and supervision units of construction safety are analyzed by the method of complete static game theory, the basic structure diagram of the game relation is established, and the 
basic flow of the game analysis is summarized. Taking the construction safety accident as a case, the game relationship between the construction unit and the safety supervision unit is analyzed dynamically, and the equilibrium strategies of the two parties are obtained. Using the Vensim software to build the construction safety supervision game model of SD, by simulation calculation and obtain the dynamic curve of strategies for the implementation of the safety rules for the construction unit, which provides theoretical reference for construction safety management research.

\section{Reference}

[1] Zeng Xueqin, Chen Jianguo. Game analysis and policy suggestion on safety supervision of construction enterprises [J]. building economy, 2013, 3 (1):15-17.

[2] Guo Na, Xiao Yuejun,Yu Shiwang. Game Analysis of engineering quality control project management based on [J]. technology, 2011, 3 (5):1-5.

[3] Ling Lingna, Su Zhenmin, Wang Xianhua. Construction based on system dynamics of construction project safety state identification model [J] Journal of safety science and technology, 2011, 3 (4): 61-62.

[4] Shen bin. Based on system dynamics of production safety supervision and effective research [J]. China Safety Science Journal, 2012, 30 (5):39-43.

[5] Cao Dongping, Wang Guangbin. Game analysis and policy suggestion on safety supervision of building production in China [J]. Building economy, 2007, 11 (5): 52-55.

[6] Sun Lingsuo, Wang Juan. The present situation and thinking about the distribution of building construction safety management responsibilities [J]. Building safety, 2009, 6 (1): 9-12.

[7] He Yao, Wang Wenqing, Xue Fei. Research on massive data mining based on cloud computing [J]. Computer technology and development, 2013, 23 (2): 69-72

[8] Ding Yan, Yang Qingping, Qian Yuming. Research on the architecture and key technologies of data mining platform based on cloud computing [J]. ZTE technology, 2013,19 (1): 53-60

[9] Li Kai, Chang Zheng. Design and implementation of parallel data mining system based on cloud computing [J]. Micro computer information, 2011, 27 (6): 121-123

[10] Sun Guanglu, Qi Haoliang. Based on online scheduling logic regression of spam filtering [J]. Journal of Tsinghua University, 2013, 53 (5):734-740

[11] Liu Botao. Research on data mining algorithms based on rough sets [J]. China western science and technology, 2011, 10 (14): 11-12 